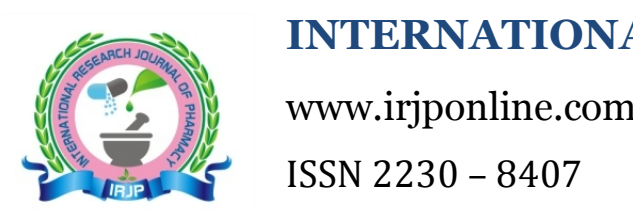

\title{
Research Article \\ SCREENING OF HERBAL LEAVES EXTRACT OF BOUGAINVILLEA GLABRA FOR DIABETIC NEPHROPATHY IN RATS
}

Pankaj Verma*, Shweta Sehrawat, Hema Chaudhary, Vikash Kumar

P. D. Memorial College of Pharmacy, Bahadurgarh, India

*Corresponding Author Email: vermapankaj1614@gmail.com

Article Received on: 21/04/16 Revised on: 10/05/16 Approved for publication: 07/06/16

\section{DOI: $10.7897 / 2230-8407.07664$}

\begin{abstract}
The main objective of this study was to investigate hypoglycemic effects of Bougainvillea glabra Leaves extract in Streptozotocin (STZ) induced hyperglycemic rats. The secondary objective of the study was to study effect of extract on biochemical parameters. Extract was prepared from leaves of Bougainvillea glabra by Soxhlet extraction method. Diabetes was induced in rats by using Streptozotocin and rats were divided into 6 groups namely control, Diabetic, treated groups at doses of $250 \mathrm{mg} / \mathrm{kg}, 500 \mathrm{mg} / \mathrm{kg}, 1000 \mathrm{mg} / \mathrm{kg}$ and Lisinopril treated group. Blood and Urine samples were collected for the estimation of Total Glycerides (TG), Serum Cholesterol, Total protein, Blood Urea Nitrogen (BUN), Serum Creatinine, Thiobarbituric acid reactive substance (TBARS) and Glutathione levels (GSH). The result showed positive effect of leaves extract to reduce hyperglycemia in treated group as compared to control group. Bougainvillea glabra extract at $1000 \mathrm{mg} / \mathrm{kg}$ dose showed efficient results.
\end{abstract}

KEYWORDS: Diabetic Nephropathy, Bougainvillea glabra, Streptozotocin, Biochemical Parameters

\section{INTRODUCTION}

Diabetes mellitus is a metabolic disorder characterised by hyperglycaemia with impaired carbohydrate, fat and protein metabolism and reduced insulin secretion ${ }^{1}$. Diabetes results in various serious complications such as diabetic retinopathy, neuropathy and nephropathy ${ }^{2}$. Loss of high amount of protein in urine during diabetes results in Diabetic nephropathy. The exact cause for it is yet unknown but it is estimated that poor control on blood sugar, high blood pressure and the people who are diabetic are mainly at risk of diabetic nephropathy ${ }^{3}$.

Diabetic nephropathy occurs as a result of an interaction between hemodynamic and metabolic factors ${ }^{4}$. Hemodynamic factors include increased systemic and intraglomerular pressure, activation of vasoactive hormone pathways including the renin angiotensin system and endothelin ${ }^{5}$. These hemodynamic pathways activate intracellular second messengers such as protein kinase $\mathrm{C}(\mathrm{PKC})$, Mitogen activated protein (MAP kinase $)^{6}$, nuclear transcription factors such as NFkB, vascular endothelial growth factor (VEGF)and result in enhanced oxidative stress, renal polyol formation (Dunlop ME) and the accumulation of advanced glycation end products (AGEs) ultimately lead to increased renal albumin permeability.

Prevalence of diabetes is increasing globally and is found maximum in developing countries like India. By the year 2010, there are about 220 million people having diabetes throughout the world. Type 2 diabetic patient in India are expected to rise from 19.4 million in 1995 to 80.5 million in $2030^{7}$.

There are numbers of synthetic drugs used for treatment of diabetic nephropathy are: ACE inhibitors (lisinopril, fosinopril), ATI receptor blockers (candesartan, telmisartan), urotensin III receptor bloker (palosura), PPAR gamma agonist (pioglitazone, rosiglitazone), rho kinase (fausudil), $\mathrm{HMG} \mathrm{CoA}$ reductase (atorvastatin, pravastatin) $^{8,9}$. These drugs have their own disadvantages like hypotension, cough and hyperkalemia caused by ACE inhibitors. Dyspepsia, muscle cramp and pharyngitis caused by ATI receptor blocker. Fluid retention, CHF and anemia caused by PPAR gamma agonist abdominal pain, dyspepsia and arthralgia caused by HMG CoA reductase etc.

Herbal medicines have gained significant importance in the last few decades and the demand to use natural products in the treatment of diabetes is increasing worldwide. Available literature shows that there are more than 400 plant species showing anti-diabetic activity.

Despite of numerous uses and pharmacological activity attributed of Bougainvillea glabra but no pharmacological activity regarding the leaves of this plant for treatment of diabetic nephropathy have been reported yet. Hence, present investigation is an attempt in this direction to assess the activity of Bougainvillea glabra leaves extract in Diabetic nephropathy. The study has been designed to investigate the effect of Bougainvillea glabra leaves extract in STZ induced diabetes.

\section{MATERIALS AND METHODS}

\section{Drug and chemicals}

The standard drug, lisinopril was procured from Astra-Zenecon, pharma Ltd, Karnataka, India. STZ was purchased from Adipogen, USA. Blood samples analyzed for blood glucose utilises one touch ultra-glucometer and strips lifescan inc. Johnson \& Jhonson company. GOD-POD kit from span diagnostic Ltd. Creatinine, triglyceride, cholesterol estimation were done using biochemical kit from ERBA Mannheim, Mumbai, India. Total protein, blood urea nitrogen, serum creatinine, TBARS and GSH parameter were estimated in pathology lab. All chemical and solvent were of reagent grade. 
Plant material

Plant material was collected randomly from local area of Bahadurgarh, Haryana and further, the identity of plant was confirmed (NHCP/NBPGR/2013-49) by National Herbarium of Cultivated Plant, National Bureau of Plant Genetic Resource, New Delhi. The plant material were dried in shade to avoid any damage to active constituents and quality samples were selected for extraction.

\section{Extraction and isolation}

Plant material is crushed in grinder and dried powder material was initially defatted with petroleum ether $\left(60-80^{\circ} \mathrm{C}\right)$ in Soxhlet apparatus for $72 \mathrm{hr}$. After successive solvent extraction, the petroleum ether extract was dried and extracted with hydroalchol (50:50) for $72 \mathrm{hr}$. The extract was filtered and percentage yield of extract was determined ${ }^{10}$.

\section{Experimental animal}

Wistar rats of either sex weighing around 200-300gm were purchased from disease free animal house of V.B Patel chest institute, Delhi. Animal were housed separately in group of 6 per cage (polycarbonate cage size: $29 \times 22 \times 14 \mathrm{~cm}$ ) under laboratory condition with alternatively light and dark cycle of 12 hour each. The animal had free access to food and water. Ethical clearance number obtained to conduct this animal experimentation protocol is PDM/CPCSEA/RES/2012/3.

\section{Induction of Diabetic nephropathy}

Diabetic nephropathy is induced by single dose of STZ (55 $\mathrm{mg} / \mathrm{kg}$, i.p), is prepared by dissolve in citrate buffer, at $\mathrm{pH} 4.5$ is given to rats. Rat were made to fasting prior to STZ administration, after approximately 6 hour, a 5\% glucose solution was offered to animal to prevent hypoglycemia the blood glucose concentration, after $72 \mathrm{hr}$ the blood sample were collected from retro orbital plexus daily for one week for evaluation of blood glucose level. Blood sample were subsequently collected from ROP on $7^{\text {th }}, 21^{\text {st }}, 35^{\text {th }}$ and $49^{\text {th }}$ day ${ }^{11}$. Rat with blood glucose level above $200 \mathrm{mg} / \mathrm{dl}$ were selected for experimental studies. After the experimental protocol all animal were sacrificed by cervical dislocation and biochemical studies were conducted on serum of experimental animal in each group.

\section{Dose Preparation and administration}

Suspension of B. glabra leaves extract was prepared for each low dose $(250 \mathrm{mg} / \mathrm{kg})$, medium dose $(500 \mathrm{mg} / \mathrm{kg})$, high dose $(1000 \mathrm{mg} / \mathrm{kg})$ resp. and lisinopril was prepared by dissolving in water.

Experimental protocol: The rats were divided in to 6 groups comprising of 6 animals in each group as follows:

Group1: Received vehicle and served as control group.

Group2: Diabetic control group, Injected with Streptozotocin STZ only.

Group 3, 4, 5: Received leaves extract of B. glabra at different doses level of 250,500 and $1000 \mathrm{mg} / \mathrm{kg}^{-1}$ body weight, respectively via oral gavage daily for 40 days, starting after 3 days of STZ injection.

Group 6: Received Standard drug Lisinopril $1 \mathrm{mg} / \mathrm{kg}^{-1}$ rat subcutaneously.

All doses were given orally once a day for 8 consecutive weeks, after completion of protocol, rats were fasted overnight prior to the blood collection.

\section{Biochemical Estimation}

Biochemical parameters such as Serum Glucose ${ }^{12}$, Total Glycerides $(\mathrm{TG})^{13}$, Serum Cholesterol ${ }^{14}$, Blood Urea Nitrogen $(\mathrm{BUN})^{15,16}$, Serum Creatinine ${ }^{17}$, Thiobarbituric acid reactive substance (TBARS) ${ }^{18}$, Total Protein ${ }^{19}$, and Glutathione levels (GSH) will be estimated using suitable kit. The blood is collected in micro-centrifuge tube in centrifuge at $4000 \mathrm{rpm}$ for $15 \mathrm{~min}$ and serum thus obtained was analysed for glucose, creatinine, cholesterol, triglyceride using commercial diagnostic kit (Erbra Mannheim, Mumbai) according to manufacturer protocol. Before the start of treatment, initial values of all parameters were estimated by same kit. Collection of urine will be done for estimating albumin levels.

\section{Statistical analysis}

The data were analysed based on t-test and one-way analysis of variance ANOVA using SPSS version 21.

\section{RESULTS}

The blood glucose levels were found to be significantly elevated in diabetic rats as compared to normal rats as showed in Table 1. Serum glucose was found to be decreased in dose dependent manner in B. glabra treated rats. Decrease in serum glucose level was highly significant on 49th day as compared to $21^{\text {st }}$ and $35^{\text {th }}$ day.

Serum triglyceride levels were found to be significantly elevated in diabetic rats as showed in Table 2. The increase in serum TG was attenuated in $B$. glabra treated groups, however this reduction was observed only during $3^{\text {rd }}$ and $5^{\text {th }}$ week of week of treatment. At the end of $7^{\text {th }}$ week no reduction in TG level was observed. Lisinopril was found to be more effective as compare to herbal extract.

In diabetic group the serum cholesterol levels were found to be elevated as showed in Table 3. Furthermore, B. glabra was found to bring back the elevated cholesterol levels in dose dependent manner. Effect was significant on $7^{\text {th }}$ week of treatment. Moreover, lisinopril shows some significant decrease in elevated serum cholesterol levels as compared to herbal extract.

Low dose of B. glabra did not shows significant improvement in BUN levels, however at high and medium dose, the effect of $B$. glabra was observed in dose dependent manner as showed in Table 4. Lisinopril exhibited a significant reduction in elevated BUN levels on $35^{\text {th }}$ and $49^{\text {th }}$ day but B. glabra also shows significant result at high dose.

Total protein levels were found to be significantly higher in diabetic rats as compared to normal group as showed in Table 5. The B. glabra treated rats showed a reduction in elevated total protein levels. The decline was significant in dose dependent manner. The reduction in total protein level in B. glabra treated rats was observed during $5^{\text {th }}$ and $7^{\text {th }}$ weeks of treatment.

A significant increase in creatinine level was observed in diabetic rats as compare to normal rats. Further, low dose of $B$. glabra leaves extract did not show any improvement in creatinine levels, however B. galbra medium and high dose shows significant decrease in creatinine level on $5^{\text {th }}$ and $7^{\text {th }}$ week of treatment as showed in Table 6. Lisinopril treated group was found to be highly significant compare to B. glabra low and medium dose. 
Table 1: Mean Serum Glucose Level (mg/dl)

\begin{tabular}{|c|c|c|c|c|c|}
\hline Groups & Treatment (Dose) & $\mathbf{7}^{\text {th }}$ Day & $\mathbf{2 1}^{\text {st }}$ Day & $\mathbf{3 5}^{\text {th }}$ Day & $\mathbf{4 9}^{\text {th }}$ Day \\
\hline 1 & Normal control & $91.83 \pm 3.06$ & $92.72 \pm 3.18$ & $91.92 \pm 3.11$ & $92.65 \pm 2.95$ \\
\hline 2 & Diabetic control & $262.12 \pm 19.76$ & $276.6 \pm 20.66^{\text {a }}$ & $285.9 \pm 21.72^{\text {a }}$ & $298.78 \pm 22.64^{\text {a }}$ \\
\hline 3 & B.glabra Low dose (250mg/dl) & $261.45 \pm 19.82$ & $274.89 \pm 19.65^{\text {a }}$ & $284.68 \pm 20.61^{\text {a }}$ & $254.85 \pm 17.54^{\text {ab }}$ \\
\hline 4 & B.glabra Med. dose (500mg/dl) & $261.61 \pm 19.46$ & $270.16 \pm 19.21^{\text {ab }}$ & $260.48 \pm 19.21^{\text {abc }}$ & $220.44 \pm 13.84^{\text {abc }}$ \\
\hline 5 & B.glabra High dose (1000mg/dl) & $260.75 \pm 18.94$ & $268.01 \pm 19.08^{\text {abc }}$ & $240.2 \pm 15.89^{\text {abcd }}$ & $184.66 \pm 13.52^{\text {abcd }}$ \\
\hline 6 & Lisinopril(1 $\mathrm{mg} / \mathrm{kg})$ & $258.61 \pm 17.96$ & $264.64 \pm 18.88^{\text {abc }}$ & $224.1 \pm 13.64^{\text {abcde }}$ & $171.78 \pm 9.67^{\text {abcde }}$ \\
\hline
\end{tabular}

All values are expressed as mean \pm S.D. $\mathrm{a}=\mathrm{P}<0.05$ vs. normal control, $\mathrm{b}=\mathrm{P}<0.05$ vs. diabetic control, $\mathrm{c}=\mathrm{P}<0.05$ vs. low dose, $\mathrm{d}=\mathrm{P}<0.05$ vs. medium dose, $\mathrm{e}=\mathrm{P}<0.05$ vs. high dose. The one way ANOVA followed by Tukey multiple comparison test.

Table 2: Mean Serum Triglyceride (mg/d)

\begin{tabular}{|c|c|c|c|c|c|}
\hline Groups & Treatment (Dose) & $7^{\text {th }}$ Day & $21^{\text {st }}$ Day & $3^{\text {th }}$ Day & $49^{\text {th }}$ Day \\
\hline 1 & Normal control & $72.09 \pm 7.89$ & $71.97 \pm 7.62$ & $72.86 \pm 7.94$ & $72.44 \pm 7.35$ \\
\hline 2 & Diabetic control & $82.6 \pm 0.391$ & $108.74 \pm 0.197^{\mathrm{a}}$ & $138.42 \pm 0.230^{\mathrm{a}}$ & $174.61 \pm 0.223^{\mathrm{a}}$ \\
\hline 3 & B.glabra Low dose $(250 \mathrm{mg} / \mathrm{dl})$ & $81.44 \pm 0.383$ & $104.62 \pm 0.163^{\mathrm{a}}$ & $134.691 \pm 0.215^{\mathrm{a}}$ & $169.54 \pm 0.219^{\mathrm{a}}$ \\
\hline 4 & B. glabra Med dose $(500 \mathrm{mg} / \mathrm{dl})$ & $78.61 \pm 0.364$ & $96.62 \pm 0.123^{\mathrm{a}}$ & $129.24 \pm 0.207^{\mathrm{ab}}$ & $160.88 \pm 0.214^{\mathrm{ab}}$ \\
\hline 5 & B.glabra High dose $(1000 \mathrm{mg} / \mathrm{dl})$ & $73.874 \pm 0.32$ & $88.22 \pm 0.118^{\mathrm{a}}$ & $117.53 \pm 0.197^{\mathrm{abc}}$ & $152.24 \pm 0.197^{\mathrm{abc}}$ \\
\hline 6 & Lisinopril $(1 \mathrm{mg} / \mathrm{kg})$ & $72.14 \pm 0.198$ & $86.911 \pm 0.097^{\mathrm{abc}}$ & $115.42 \pm 0.192^{\mathrm{abc}}$ & $151.64 \pm 0.182^{\mathrm{abc}}$ \\
\hline
\end{tabular}

All values are expressed as mean \pm S.D. $\mathrm{a}=\mathrm{P}<0.05$ vs. normal control, $\mathrm{b}=\mathrm{P}<0.05$ vs. diabetic control, $\mathrm{c}=\mathrm{P}<0.05$ vs. low dose, $\mathrm{d}=\mathrm{P}<0.05$ vs. medium dose, $\mathrm{e}=\mathrm{P}<0.05$ vs. high dose. The one way ANOVA followed by Tukey multiple comparison test.

Table 3: Mean Serum Cholesterol (mg/dl)

\begin{tabular}{|c|c|c|c|c|c|}
\hline Groups & Treatment (Dose) & $7^{\text {th }}$ Day & $21^{\text {st }}$ Day & $35^{\text {th }}$ Day & $49^{\text {th }}$ Day \\
\hline 1 & Normal control & $61.69 \pm 0.256$ & $62.94 \pm 0.291$ & $61.54 \pm 0.234$ & $62.47 \pm 0.258$ \\
\hline 2 & Diabetic control & $60.69 \pm 0.317$ & $125.24 \pm 0.342^{\mathrm{a}}$ & $141.99 \pm 0.383^{\mathrm{a}}$ & $160.32 \pm 0.385^{\mathrm{a}}$ \\
\hline 3 & B.glabra Low dose $(250 \mathrm{mg} / \mathrm{kg})$ & $59.49 \pm 0.291$ & $115.66 \pm 0.336^{\mathrm{a}}$ & $120.98 \pm 0.374^{\mathrm{ab}}$ & $135.61 \pm 0.368^{\mathrm{ab}}$ \\
\hline 4 & B.glabra med dose $(500 \mathrm{mg} / \mathrm{kg})$ & $58.94 \pm 0.297$ & $110.94 \pm 0.321^{\mathrm{ab}}$ & $117.64 \pm 0.361^{\mathrm{ab}}$ & $127.22 \pm 0.379^{\mathrm{abc}}$ \\
\hline 5 & B.glabra high dose $(1000 \mathrm{mg} / \mathrm{kg})$ & $57.22 \pm 0.271$ & $99.88 \pm 0.317^{\mathrm{abc}}$ & $104.6 \pm 0.338^{\text {abcd }}$ & $110.44 \pm 0.341^{\text {abcd }}$ \\
\hline 6 & Lisinopril $(1 \mathrm{mg} / \mathrm{kg})$ & $57.08 \pm 0.232$ & $85.66 \pm 0.301^{\mathrm{abcd}}$ & $99.121 \pm 0.302^{\text {abcd }}$ & $101.97 \pm 0.368^{\text {abcde }}$ \\
\hline
\end{tabular}

All values are expressed as mean \pm S.D. $\mathrm{a}=\mathrm{P}<0.05$ vs. normal control, $\mathrm{b}=\mathrm{P}<0.05$ vs. diabetic control, $\mathrm{c}=\mathrm{P}<0.05$ vs. low dose, $\mathrm{d}=\mathrm{P}<0.05$ vs. medium dose, $\mathrm{e}=\mathrm{P}<0.05$ vs. high dose. The one way ANOVA followed by Tukey multiple comparison test.

Table 4: Mean Serum BUN Levels $(\mathrm{mg} / \mathrm{dl})$

\begin{tabular}{|c|c|c|c|c|c|}
\hline Groups & Treatment (Dose) & $7^{\text {th }}$ Day & $\mathbf{2 1}^{\text {st }}$ Day & 35 $^{\text {th }}$ Day & $\mathbf{4 9}^{\text {th }} \mathbf{D a y}$ \\
\hline 1 & Normal control & $16.97 \pm 14.84$ & $17.89 \pm 14.92$ & $16.23 \pm 14.73$ & $17.24 \pm 14.91$ \\
\hline 2 & Diabetic control & $24.64 \pm 0.423$ & $26.87 \pm 0.274^{\mathrm{a}}$ & $28.96 \pm 0.320^{\mathrm{a}}$ & $30.64 \pm 0.364^{\mathrm{a}}$ \\
\hline 3 & B.glabra Low dose(250mg/kg) & $24.68 \pm 0.425$ & $24.67 \pm 0.423^{\mathrm{a}}$ & $25.34 \pm 0.342^{\mathrm{a}}$ & $26.98 \pm 0.283^{\mathrm{ab}}$ \\
\hline 4 & B.glabra med dose $(500 \mathrm{mg} / \mathrm{kg})$ & $24.51 \pm 0.419$ & $24.389 \pm 0.408^{\mathrm{a}}$ & $24.85 \pm 0.452^{\mathrm{a}}$ & $25.64 \pm 0.294^{\mathrm{ab}}$ \\
\hline 5 & B.glabra high dose $(1000 \mathrm{mg} / \mathrm{kg})$ & $24.49 \pm 0.417$ & $23.89 \pm 0.399^{\mathrm{ab}}$ & $23.99 \pm 0.419^{\mathrm{ab}}$ & $24.87 \pm 0.385^{\mathrm{ab}}$ \\
\hline 6 & Lisinopril $(1 \mathrm{mg} / \mathrm{kg})$ & $24.378 \pm 0.408$ & $23.21 \pm 0.394^{\mathrm{ab}}$ & $23.516 \pm 0.402^{\mathrm{ab}}$ & $22.994 \pm 0.216^{\mathrm{abc}}$ \\
\hline
\end{tabular}

All values are expressed as mean \pm S.D. $\mathrm{a}=\mathrm{P}<0.05$ vs. normal control, $\mathrm{b}=\mathrm{P}<0.05$ vs. diabetic control, $\mathrm{c}=\mathrm{P}<0.05$ vs. low dose, $\mathrm{d}=\mathrm{P}<0.05$ vs. medium dose, $\mathrm{e}=\mathrm{P}<0.05$ vs. high dose. The one way ANOVA followed by Tukey multiple comparison test.

Table 5: Mean Serum Total Protein $(\mathrm{mg} / \mathrm{ml})$

\begin{tabular}{|c|c|c|c|c|c|}
\hline Groups & Treatment (Dose) & $7^{\text {th }}$ Day & $21^{\text {st }}$ Day & $35^{\text {th }}$ Day & $49^{\text {th }}$ Day \\
\hline 1 & Normal control & $5.34 \pm 0.0387$ & $5.41 \pm 0.0394$ & $5.38 \pm 0.0391$ & $5.40 \pm 0.0394$ \\
\hline 2 & Diabetic control & $20.67 \pm 0.299$ & $42.78 \pm 0.423^{\mathrm{a}}$ & $84.66 \pm 11.102^{\mathrm{a}}$ & $106.94 \pm 0.342^{\mathrm{a}}$ \\
\hline 3 & B.glabra Low dose $(250 \mathrm{mg} / \mathrm{kg})$ & $19.61 \pm 0.295$ & $35.94 \pm 0.356^{\mathrm{ab}}$ & $72.28 \pm 0.287^{\mathrm{ab}}$ & $86.88 \pm 0.3317^{\mathrm{ab}}$ \\
\hline 4 & B.glabra med dose $(500 \mathrm{mg} / \mathrm{kg})$ & $18.42 \pm 0.284$ & $30.66 \pm 0.171^{\mathrm{ab}}$ & $63.61 \pm 0.287^{\mathrm{abc}}$ & $72.12 \pm 0.263^{\mathrm{abc}}$ \\
\hline 5 & B.glabra high dose $(1000 \mathrm{mg} / \mathrm{kg})$ & $17.82 \pm 0.276$ & $24.24 \pm 0.351^{\mathrm{abcd}}$ & $54.73 \pm 0.154^{\text {abcd }}$ & $60.24 \pm 11.16^{\mathrm{abcd}}$ \\
\hline 6 & Lisinopril $(1 \mathrm{mg} / \mathrm{kg})$ & $17.22 \pm 0.270$ & $21.11 \pm 0.301^{\mathrm{abcd}}$ & $43.84 \pm 0.146^{\text {abcde }}$ & $53.64 \pm 1.007^{\mathrm{abcde}}$ \\
\hline
\end{tabular}

All values are expressed as mean \pm S.D. $\mathrm{a}=\mathrm{P}<0.05$ vs. normal control, $\mathrm{b}=\mathrm{P}<0.05$ vs. diabetic control, $\mathrm{c}=\mathrm{P}<0.05$ vs. low dose, $\mathrm{d}=\mathrm{P}<0.05$ vs. medium dose, $\mathrm{e}=\mathrm{P}<0.05$ vs. high dose. The one way ANOVA followed by Tukey multiple comparison test.

Table 6: Mean Serum Creatinine Levels $(\mathrm{mg} / \mathrm{dl})$

\begin{tabular}{|c|c|c|c|c|c|}
\hline Groups & Treatment days & $\mathbf{7}^{\text {th }}$ day & $\mathbf{2 1}^{\text {st }}$ day & $\mathbf{3 5}^{\text {th }}$ day & $\mathbf{4 9}^{\text {th }}$ day \\
\hline 1 & Normal control & $0.81 \pm 0.012$ & $0.79 \pm 0.0193$ & $0.81 \pm 0.0121$ & $0.78 \pm 0.0191$ \\
\hline 2 & Diabetic control & $1.278 \pm 0.0032$ & $1.46 \pm 0.0037^{\mathrm{a}}$ & $1.87 \pm 0.0020^{\mathrm{a}}$ & $2.08 \pm 0.0317^{\mathrm{a}}$ \\
\hline 3 & B.glabra Low dose $(250 \mathrm{mg} / \mathrm{kg})$ & $1.225 \pm 0.00147$ & $1.38 \pm 0.0012^{\mathrm{ab}}$ & $1.76 \pm 0.0918^{\mathrm{ab}}$ & $1.97 \pm 0.0346^{\mathrm{ab}}$ \\
\hline 4 & B.glabra med dose $(500 \mathrm{mg} / \mathrm{kg})$ & $1.198 \pm 0.097$ & $1.33 \pm 0.097^{\mathrm{ab}}$ & $1.56 \pm 0.0902^{\mathrm{abc}}$ & $1.82 \pm 0.0318^{\mathrm{abc}}$ \\
\hline 5 & B.glabra high dose $(1000 \mathrm{mg} / \mathrm{kg})$ & $1.165 \pm 0.083$ & $1.31 \pm 0.092^{\mathrm{abc}}$ & $1.38 \pm 0.087^{\mathrm{abcd}}$ & $1.51 \pm 0.826^{\mathrm{abcd}}$ \\
\hline 6 & Lisinopril( $1 \mathrm{mg} / \mathrm{kg})$ & $1.145 \pm 0.71$ & $1.30 \pm 0.023^{\mathrm{abc}}$ & $1.312 \pm 0.082^{\mathrm{abcd}}$ & $1.27 \pm 0.676^{\mathrm{abcde}}$ \\
\hline
\end{tabular}

All values are expressed as mean \pm S.D. $\mathrm{a}=\mathrm{P}<0.05$ vs. normal control, $\mathrm{b}=\mathrm{P}<0.05$ vs. diabetic control, $\mathrm{c}=\mathrm{P}<0.05$ vs. low dose, $\mathrm{d}=\mathrm{P}<0.05$ vs. medium dose, $\mathrm{e}=\mathrm{P}<0.05$ vs. high dose. The one way ANOVA followed by Tukey multiple comparison test. 
Table 7: Mean Serum Thiobarbituric acid reactive substance (TBARS) (nmol/mg)

\begin{tabular}{|c|c|c|c|c|c|}
\hline Groups & Treatment (Dose) & $7^{\text {th }}$ Day & $21^{\text {st }}$ Day & $3^{\text {th }}$ Day & $49^{\text {th }}$ Day \\
\hline 1 & Normal control & $0.62 \pm 0.03$ & $0.69 \pm 0.039$ & $0.71 \pm 0.0467$ & $0.68 \pm 0.032$ \\
\hline 3 & B.Glabra Low dose(250mg/dl) & $0.83 \pm 0.0113$ & $0.917 \pm 0.005^{\mathrm{a}}$ & $1.2 \pm 0.0022^{\mathrm{a}}$ & $1.63 \pm 0.0265^{\mathrm{ab}}$ \\
\hline 5 & B. Glabra High dose $(1000 \mathrm{mg} / \mathrm{dl})$ & $0.76 \pm 0.0179$ & $0.85 \pm 0.0114^{\mathrm{abc}}$ & $0.99 \pm 0.0021^{\mathrm{abc}}$ & $1.36 \pm 0.0423^{\mathrm{abcd}}$ \\
\hline 6 & Lisinopril $(1 \mathrm{mg} / \mathrm{kg})$ & $0.73 \pm 0.0175$ & $0.82 \pm 0.0196^{\mathrm{abcd}}$ & $0.91 \pm 0.0195^{\text {abcde }}$ & $1.05 \pm 0.023^{\mathrm{abcde}}$ \\
\hline
\end{tabular}

All values are expressed as mean \pm S.D. $\mathrm{a}=\mathrm{P}<0.05$ vs. normal control, $\mathrm{b}=\mathrm{P}<0.05$ vs. diabetic control, $\mathrm{c}=\mathrm{P}<0.05$ vs. low dose, $\mathrm{d}=\mathrm{P}<0.05$ vs. medium dose, $\mathrm{e}=\mathrm{P}<0.05$ vs. high dose. The one way ANOVA followed by Tukey multiple comparison test.

Table 8: Mean Serum Glutathione level (nmol/mg)

\begin{tabular}{|c|c|c|c|c|c|}
\hline Groups & Treatment (Dose) & $7^{\text {th }}$ Day & $21^{\text {st }}$ Day & $35^{\text {th }}$ Day & $49^{\text {th }}$ Day \\
\hline 1 & Normal control & $21.54 \pm 0.49$ & $21.52 \pm 0.206$ & $21.98 \pm 0.218$ & $21.55 \pm 0.208$ \\
\hline 2 & Diabetic control & $19.47 \pm 0.294$ & $17.51 \pm 0.137^{\mathrm{a}}$ & $14.94 \pm 0.344^{\mathrm{a}}$ & $12.60 \pm 0.69^{\mathrm{a}}$ \\
\hline 3 & B.glabra Low dose $(250 \mathrm{mg} / \mathrm{kg})$ & $19.74 \pm 0.295$ & $17.73 \pm 0.139^{\mathrm{a}}$ & $14.99 \pm 0.348^{\mathrm{a}}$ & $12.64 \pm 0.692^{\mathrm{a}}$ \\
\hline 4 & B.glabra med dose $(500 \mathrm{mg} / \mathrm{kg})$ & $20.97 \pm 0.223$ & $17.91 \pm 0.142^{\mathrm{a}}$ & $15.78 \pm 0.356^{\mathrm{abc}}$ & $12.83 \pm 0.72^{\mathrm{a}}$ \\
\hline 5 & B.glabra high dose $(1000 \mathrm{mg} / \mathrm{kg})$ & $21.14 \pm 0.237$ & $18.82 \pm 0.267^{\mathrm{abc}}$ & $15.876 \pm 0.387^{\mathrm{abc}}$ & $13.94 \pm 0.836^{\text {abcd }}$ \\
\hline 6 & Lisinopril $(1 \mathrm{mg} / \mathrm{kg})$ & $21.85 \pm 0.285$ & $19.23 \pm 0.292^{\mathrm{abcd}}$ & $15.94 \pm 0.392^{\mathrm{abcd}}$ & $16.68 \pm 0.76^{\text {abcde }}$ \\
\hline
\end{tabular}

All values are expressed as mean \pm S.D. $\mathrm{a}=\mathrm{P}<0.05$ vs. normal control, $\mathrm{b}=\mathrm{P}<0.05$ vs. diabetic control, $\mathrm{c}=\mathrm{P}<0.05$ vs. low dose, $\mathrm{d}=\mathrm{P}<0.05$ vs. medium dose, $\mathrm{e}=\mathrm{P}<0.05$ vs. high dose. The one way ANOVA followed by Tukey multiple comparison test.

In comparison to normal control rat, TBARS levels were found to be decrease in dose dependent manner in B. glabra treated rats and TBARS levels were highly significant on $5^{\text {th }}$ and $7^{\text {th }}$ week of treatment as compare to $3^{\text {rd }}$ week of treatment as showed in Table 7. However lisinopril shows significant decrease in TBARS level on $7^{\text {th }}$ week of treatment as compared to $B$. glabra leaves extract treated group.

GSH levels were found to be decreased in diabetic group as showed in Table 8. The increase in GSH level was observed in $B$. glabra leaves extract treated group, however this improvement was observed in $5^{\text {th }}$ and $7^{\text {th }}$ week as compared to $1^{\text {st }}$ and $3^{\text {rd }}$ week of treatment. Standard drug treatment with lisinopril was found to be more effective as compare to $B$. glabra leaves extract.

\section{DISCUSSION}

The present study is an attempt made to check the effect of $B$. glabra leaves extract in STZ induced diabetic rats. Diabetes mellitus is clinically and genetically heterogenous group of metabolic disorder manifested by abnormally high level of glucose in blood. Diabetes after long time develops severe complication like diabetic retinopathy, diabetic neuropathy, diabetic micro-angiopathy and diabetic nephropathy etc.

The increase in levels of serum creatinine, BUN and total protein has been documented to be index of renal dysfunction. In present study, these parameter were elevated in diabetic group but on treatment with $B$. glabra at different doses, decreasing levels of serum creatinine, BUN and total protein were observed.

Hyperlipidemia is an independent risk factor for progression of diabetes induced nephropathy but treatment with $B$. glabra leaves extract attenuates these lipid levels. Increase in renal TBARS \& decrease in GSH levels are considered as index of development of Oxidative Stress which result in renal cell injury \& ECM deposition. So, treatment with herbal extract results in decreased oxidative stress due to their antioxidant activity which improves GSH levels \& decreases TBARS levels. Direct renoprotective action of bougainvillea may be due to their antioxidant property on diabetic kidney. Moreover, the effect of Bougainvillea extract is observed in reducing creatinine, total protein, glucose, TG and cholesterol ${ }^{20}$.
Diabetic animal treated with this extract shows less significant result in case of cholesterol and triglyceride but significant result was observed in case of BUN, creatinine, and total protein i.e. extract decreases creatinine, BUN and total protein level. Blood glucose levels were elevated in diabetic rat but extract treated group shows decreased glucose levels to some extent.

Present study revealed that administration of $B$. glabra leaves extract shows nephroprotective activity at the dose of $1000 \mathrm{mg} / \mathrm{kg}$ as compared to other treatment dose found effective in attenuating diabetic nephropathy. B. glabra have nephroprotective activity due to presence of various chemical constituent like terpenes, pinitol, flavonoids, tannin, alkaloids and saponin in it.

\section{ACKNOWLEDGEMENT}

The authors are grateful to Chairman/Management of PDM Group of Institutions; College of Pharmacy for providing proper support and guidance.

\section{REFERENCES}

1. Batra S, Nagori BP, Batra N. Medicinal Plants of Rajasthan (India) with anti-diabetic potential. Int Res J Pharm 2011; 2(3): 1-7.

2. Flower MJ. Microvascular and Macrovascular complication of diabetes. Clinical Diabetes 2008; 26: 77-82.

3. Inzucchi SE, Sherwin RS, Goldman L, Ausiello D. Diabetes Mellitus. Cecil Textbook of Medicine; 2007.

4. Cooper M. Interaction of metabolic and hemodynamic factors in mediating experimental diabetic nephropathy. Diabetologia 2001; 44 (11): 1957-72.

5. Hargrove GM, Wong JD. Diabetes mellitus increases endothelin1 gene transcription in rat kidney. Kidney International 2000; 58 (4): 1534-45.

6. Haneda M. Mitogenactivated protein kinase cascade is activated in glomeruli of diabetic rats and glomerular mesangial cells cultured under high glucose conditions. Diabetes 1997; 46 (5): 847-53.

7. Agarwal SK, Dash SC. Spectrum of Renal Disease in Indian Adults. Journal of the association of physician of India 2000; 48: 594-00. 
8. Kobayashi N, Honda T, Yoshida K. Critical role of bradykinin NOS and oxidative stressLOX pathway in cardiovascular remodelling under chronic angiotensin converting enzyme inhibition. Asian journal of Experimental Sciences 2006; 87: 92100.

9. Endres M, Laufs U. Effects of statins on endothelium and signalling mechanisms. Journal of Pharmacology and Toxicology 2004; 35: 2708-11.

10. Harborne JB. Method of extraction and isolation. In: phytochemical method, chapman and Hall, London. Journal of Pharmacy and Pharmacology 1998; 60-66.

11. Arora MK, Reddy K, Balakumar P. ACE inhibitor inhibit the formation of tubulointerstitial fibrosis in type 2 diabetic rats. Journal of American Society Nephrology 2006; 17: 1362-72.

12. Trinder P. Mono-reagent Enzymatic Glucose. Analytical clinical chemistry. Philadelphia: Saunder company; 1969. p. 24-27.

13. Fossati P, Lorenzo P. Serum Triglyceride determined colorimetrically with an enzyme that produces hydrogen peroxide. Clinical chemistry 1982; 28: 2077-80.

14. Allain CC, Poon LS, Chan CSG, Richmond W, Fu PC. Ezymatic Determination of total serum cholesterol. Clinical Chemistry 1974; 20(4): 470-75.
15. Fischbach FT, Dunning MB. A Manual of Laboratory and Diagnostic Tests. USA: Lippincott Williams \& Wilkins; 2004.

16. Checchio LM, Como AJ. Electrolytes, BUN, creatinine: who's at risk. Annal of Emergency Medicine 1986; 15(3): 363-66.

17. Botham KM, Mayes PA. Estimation of Serum Creatinine. Harper's illustrated biochemistry. New York: The McGraw -Hill Companies inc; 2006. p. 217-47.

18. Ohkawa H, Ohishi N, Yagi K. Assay for lipid peroxides in animal tissue by thiobarbituric acid reaction. Analytical Biochemistry 1979; 95: 351-358.

19. Cannon DC, Harper \& Row, Henry RJ. Proteins in clinical chemistry-Principles

Technics. Analytical Biochemistry 1974; 2: 422-431.

20. Yakubu MT, Afolayan AJ. Effect of Bougainvillea glabra on haematological and serum lipid profile of male wistar rats. Indian Journal of Experimental Biology 2007; 45: 617 619.

\section{Cite this article as:}

Pankaj Verma, Shweta Sehrawat, Hema Chaudhary, Vikash Kumar. Screening of herbal leaves extract of Bougainvillea glabra for diabetic nephropathy in rats. Int. Res. J. Pharm. 2016;7(6):58-62 http://dx.doi.org/10.7897/2230-8407.07664

Source of support: Nil, Conflict of interest: None Declared

Disclaimer: IRJP is solely owned by Moksha Publishing House - A non-profit publishing house, dedicated to publish quality research, while every effort has been taken to verify the accuracy of the content published in our Journal. IRJP cannot accept any responsibility or liability for the site content and articles published. The views expressed in articles by our contributing authors are not necessarily those of IRJP editor or editorial board members. 